Proceeding

\title{
Surgical complications after cesarean delivery and frequently indications
}

Volume 5 Issue 5 - 2016

\section{Proceeding}

Objectives: The purpose of this study is to determine complications following cesarean delivery, to identify risk factors for surgical site infection and also to evaluate frequently indications for cesarean delivery.

Material and Methods: We have studied a cohort of 219 women who were delivered by cesarean section in our clinic. Complications following cesarean delivery were determined during hospitalization of patients, review of laboratory results and follow-up after discharge.

Results: Surgical complications after cesarean delivery were rare. Only $1 \%$ of patients were febrile, while $8.2 \%$ had wound infection following delivery. Of these $7.8 \%$ were with superficial wound infection, and with deep tissue infection was $0.5 \%$. No patients had systemic infections. Surgical site infections are most often found in patients with premature rupture of membranes (PROM), and patients with emergency caesarean section, OR 3.715 (95\% CI 1.347 to 10.248 ) and OR 2.236 (95\% CI 0.796 to 6.282 ), respectively. Repeated cesarean was indication for caesarean section in $42 \%$ of cases, while PIH syndrome had a share of $13.2 \%$, cephalopelvic disproportion $11.4 \%$, PROM $10 \%$, fetal asphyxia $9.6 \%$, breech presentation $8.2 \%$, intrauterine growth restriction $7.3 \%$, dystocia was indication in $5.9 \%$ and preeclampsia/eclampsia in $4.6 \%$ of patients. Cases with concomitant morbidity were found in $26.9 \%$ of patients examined.

\section{Conclusion}

Complications after caesarean delivery were rare. Surgical site infections are most often found in patients with PROM and patients who had an emergency caesarean delivery.

\author{
Fehmi Ahmeti,' H Derguti,' B Krasniqi,' E \\ Ahmeti $^{2}$ \\ 'University Clinical Centre, Prishtina, Kosovo \\ 2University of Prishtina, Kosovo
}

Correspondence: Fehmi Ahmeti, Obstetrics and Gynecology Clinic, University Clinical Centre, Prishtina 10000, Kosovo, Tel 37744I60986, Email fehmiahmeti@hotmail.com

Received: February 07, 2016 | Published: November 10, 2016

\section{Acknowledgments}

None.

\section{Conflicts of interest}

None. 